\title{
Gasoline analysis by headspace mass spectrometry and near infrared spectroscopy
}

\author{
Marta Ferreiro-González $^{\text {b }}$, Jesús Ayuso ${ }^{\mathrm{b}}$, José A. Álvarez ${ }^{\mathrm{b}}$, Miguel Palma ${ }^{\mathrm{a}, *}$, Carmelo G. Barroso $^{\mathrm{a}}$ \\ a Department of Analytical Chemistry, Faculty of Sciences, University of Cadiz, Apartado 40, 11510 Puerto Real, Cádiz, Spain \\ ${ }^{\mathrm{b}}$ Department of Physical Chemistry, Faculty of Sciences, University of Cadiz, Apartado 40, 11510 Puerto Real, Cádiz, Spain
}

\section{H I G H L I G H T S}

- HS-MS and NIRS allow for gasoline samples discrimination.

- No solvents are need for the HS-MS analyses nor for NIRS analyses, no wastes are produced.

- HS-MS method produced similar or better results than NIRS for the discrimination among gasoline samples.

\section{A R T I C L E I N F O}

\section{Article history:}

Received 30 October 2014

Received in revised form 7 March 2015

Accepted 10 March 2015

Available online 19 March 2015

\section{Keywords:}

Gasoline

Discrimination

Headspace-mass spectrometry

Research octane number

Forensic sciences

\begin{abstract}
A B S T R A C T
Headspace mass spectrometry and near infrared spectroscopy in combination with chemometric tools, including hierarchical cluster analysis and linear discriminant analysis, have been used for the classification of gasoline according to the research octane number. Volatile components were studied by headspace mass spectrometry whereas the whole gasoline samples were studied by near infrared spectroscopy. Sample pretreatment was not required for either analysis. A set of 60 samples belonging to two different research octane numbers (95\# and 98\#) was analyzed by both techniques. The best results were obtained on using linear discriminant analysis, which allowed a full discrimination of the gasoline samples using only four $m / z$ ratios $(46,59,95$ and 98 ) in the case of headspace mass spectrometry and three regions (below $1000 \mathrm{~nm}, 1400-1500 \mathrm{~nm}$ and $1600 \mathrm{~nm}$ ) in the case of near infrared spectroscopy.
\end{abstract}

(c) 2015 Elsevier Ltd. All rights reserved.

\section{Introduction}

A typical gasoline is predominantly a mixture of volatile hydrocarbons such as paraffins (alkanes), naphthenes (cycloalkenes) and olefins (alkenes), although it also contains compounds with oxygen, nitrogen or sulfur. Automotive gasoline is one of the most refined products and the specific process involved in gasoline production defines the final chemical composition of gasoline fractions and the resulting gasoline quality [1]. The production of gasoline must meet a variety of specifications and the quality of the resulting gasoline must satisfy the national standard requirements.

Several different gasoline types can currently be found in the market, with at least two different types available in most countries. Different countries use different research octane number

\footnotetext{
* Corresponding author at: Department of Analytical Chemistry, Faculty of Sciences, University of Cadiz, Campus Universitario, 11510 Puerto Real, Spain. Tel.: +34 956016360; fax: +34956016460.

E-mail address: miguel.palma@uca.es (M. Palma).
}

levels and most employ 93-95 for the lowest level and 96-98 for the highest. The gasoline market in Spain and most European countries includes 2 different types of gasoline that are differentiated by the RON: 95\# and 98\# [2]. RON is a measure of a fuel's resistance to knock or to ignite prematurely and it is determined using the Co-operative Fuel Research (CFR) engine according to ASTM D2699-08 [3].

The identification and discrimination of gasoline types is of primary importance for numerous reasons, the most important of which are quality control, ecological monitoring, and forensic science. In relation to forensic sciences, accelerants such as ignitable liquids are commonly used to start fires in arson attacks. The most commonly used ignitable liquids are petroleum-based products like gasoline because they are easy to obtain. In some cases, traces of gasoline or other ignitable liquids remain at the fire scene and these can be matched to samples that are collected in a database or associated with a particular suspect [4]. From a chemical point of view, gasoline and other ignitable liquids have different fingerprints and these help to discriminate between different 
liquids and allow the investigator to establish links between a suspect and a fire scene $[5,6]$. For this reason, in some laboratories work is being carried out to develop databases and these include the Ignitable Liquid Reference Collection (ILRC) at NCFS in The United States [7]. In fuel spill investigation it is also important to identify the fuel source - an activity that is not always straightforward $[8,9]$. It is also worth mentioning that there are several forms of illegal practice within the fuel market. For instance, the addition of lower price components, such as solvents, to gasoline due to the large difference in the taxation of gasoline and solvents $[10,11]$. As a consequence, there is a strong need to develop analytical techniques for gasoline characterization.

The most widely used analytical methods for the classification of these liquids are gas-chromatography coupled with an FID $[12,13]$ or MS detector $[4,14,15]$. Indeed, the American Society for Testing and Materials (ASTM) has approved GC-MS as the standard method for the analysis of ignitable liquids even in fire debris [16]. However, the application of numerous different spectroscopic techniques is also described in the literature and these include FT-IR [10,11,17], NIR [18,19] and MIR [20-23] or Raman [24] spectroscopy combined with multivariate analysis for the characterization and quality control of fuels. These spectroscopic methods have several advantages over chromatographic techniques, e.g. the sample requires little or no preparation, the sample is not destroyed, reagents are not required, the equipment is relatively cheap and the analysis is quick.

In contrast to the above, compared with the chromatographic techniques, these spectroscopic techniques do have some drawbacks, mainly because spectroscopic signals cannot be assigned to specific compounds in the gasoline samples, meaning that they provide little information about individual components.

Since gasoline samples and other ignitable liquids contain large amounts of volatile organic compounds, VOCs, headspace-based techniques such as headspace mass spectrometry (HS-MS) have potential in this field. To date, most applications of these techniques are related to food and beverage, cigarette, pharmaceutical and paper industries, biotechnology, medicine, wastewater treatment plants and security companies. In many of these applications this application is also known as an electronic nose (EN).

Primary results on the application of an EN based on sensors for the classification of gasoline, heating oil and diesel oil can be found in the literature [25].

This technique does have specific advantages. Apart from the speed of the analysis, the fact that the sample does not require preparation, the absence of residues because solvents are not used, this technique also has high sensitivity, good accuracy, low cost, it is easy to handle for routine analysis and the required sample volume is very small. Most of these advantages are also provided by the NIRS systems [26], which have previously been applied for gasoline characterization $[20,21]$ and related samples as biodiesel [27].

In the work described here, a total of 60 gasoline samples provided by different Spanish refineries and belonging to 2 different RON (95\# and 98\#) were analyzed by both HS-MS and NIRS. The results obtained with both techniques were compared. A chemometric study was carried out in order to obtain a discrimination method for both sets of samples.

\section{Materials and methods}

\subsection{Gasoline samples}

60 gasoline samples were purchased from different gas stations corresponding to different Spanish refineries. The samples corresponded to two different types characterized by the RON: 30 samples from 95\# and 30 samples from 98\#.

\subsection{Acquisition of HS-MS spectra}

Gasoline analysis was performed with an HS-MS Alpha Moss (Toulouse, France) system that consisted of an HS 100 static headspace autosampler and an $\alpha$ Kronos quadrupole mass spectrometer (MS). The samples were contained in $10 \mathrm{~mL}$ sealed vials and each sample was placed in the autosampler oven to be heated and agitated in order to generate the headspace. Headspace was finally taken from the vial using a gas syringe and injected into the mass spectrometer detector. The gas syringe was heated above the sample temperature $\left(+5^{\circ} \mathrm{C}\right)$ to avoid condensation phenomenon. Between each sample injection, the gas syringe was flushed with carrier gas (nitrogen) to avoid cross-contamination.

The experimental conditions for the headspace sampler were as follows [28]: sample volume of $80 \mu \mathrm{L}$, incubation temperature $145^{\circ} \mathrm{C}$, incubation time $10 \mathrm{~min}$, agitation speed $500 \mathrm{rpm}$, syringe type $5 \mathrm{~mL}$, syringe temperature $150{ }^{\circ} \mathrm{C}$, flushing time $120 \mathrm{~s}$, fill speed $100 \mu \mathrm{L} / \mathrm{s}$, injection volume $4.5 \mathrm{~mL}$ and injection speed $75 \mu \mathrm{L} / \mathrm{s}$. The carrier gas was nitrogen and $2 \mu \mathrm{L}$ of TBPFA (perfluorotributylamine) were added to all samples as an internal standard. The total time per sample was approximately $10 \mathrm{~min}$. The components in the headspace of the vials were passed directly to the mass detector without any chromatographic separation or sample pre-treatment. In this way, for any given measurement, the resulting mass spectrum gives a fingerprint of the gasoline. MS spectra were recorded in the range $m / z$ 45-200. Instrument control was achieved using RGA (Residual Gas Analysis software package) and Alpha Soft 7.01 software.

\subsection{Acquisition of NIR spectra}

The 60 samples were also analyzed by NIR spectrometry. NIR spectra were collected in an AvasSpec-NIR 256-1.7 equipped with a tungsten halogen lamp and a transmittance probe with a path length of $10 \mathrm{~mm}$. The spectra were acquired at room temperature. Transmittance values in the range of 891-1812 nm were acquired with a resolution of $3.4 \mathrm{~nm}$.

\subsection{Data analysis and software}

Multivariate analysis of the data, which included hierarchical cluster analysis (HCA) and linear Discriminant Analysis (LDA), was performed using the statistical computer package SPSS 17.0. (Chicago, IL, USA) [29].

\section{Results and discussion}

\subsection{HS-MS chemometric Study}

The whole set of 60 gasoline samples (Gas 95\# and Gas 98\#) were analyzed by HS-MS. All mass spectra were normalized at an $m / z$ of 131 , which is the significant $m / z$ of the internal standard (TBPFA), because previous analyses of the gasoline samples did not produce a signal at $m / z 131$. The mass spectra of the 60 gasoline samples are shown in Fig. 1. The spectra for gasoline 95\# and 98\# appear to be quite similar and it is therefore very difficult to distinguish between the two types of gasoline based only on their HS-MS spectra. This finding demonstrates the need to use chemometric techniques for the classification of these samples.

An exploratory chemometric technique was initially carried out in order to check for a general distribution of the gasoline samples. An HCA (hierarchical cluster analysis) was applied using all of the $\mathrm{m} / \mathrm{z}$ values $(45-200 \mathrm{~m} / z$ ) as variables to form groups. The results of the cluster analysis are shown in a dendrogram in Fig. 2, in which all of the samples are listed, and this indicates the level of 


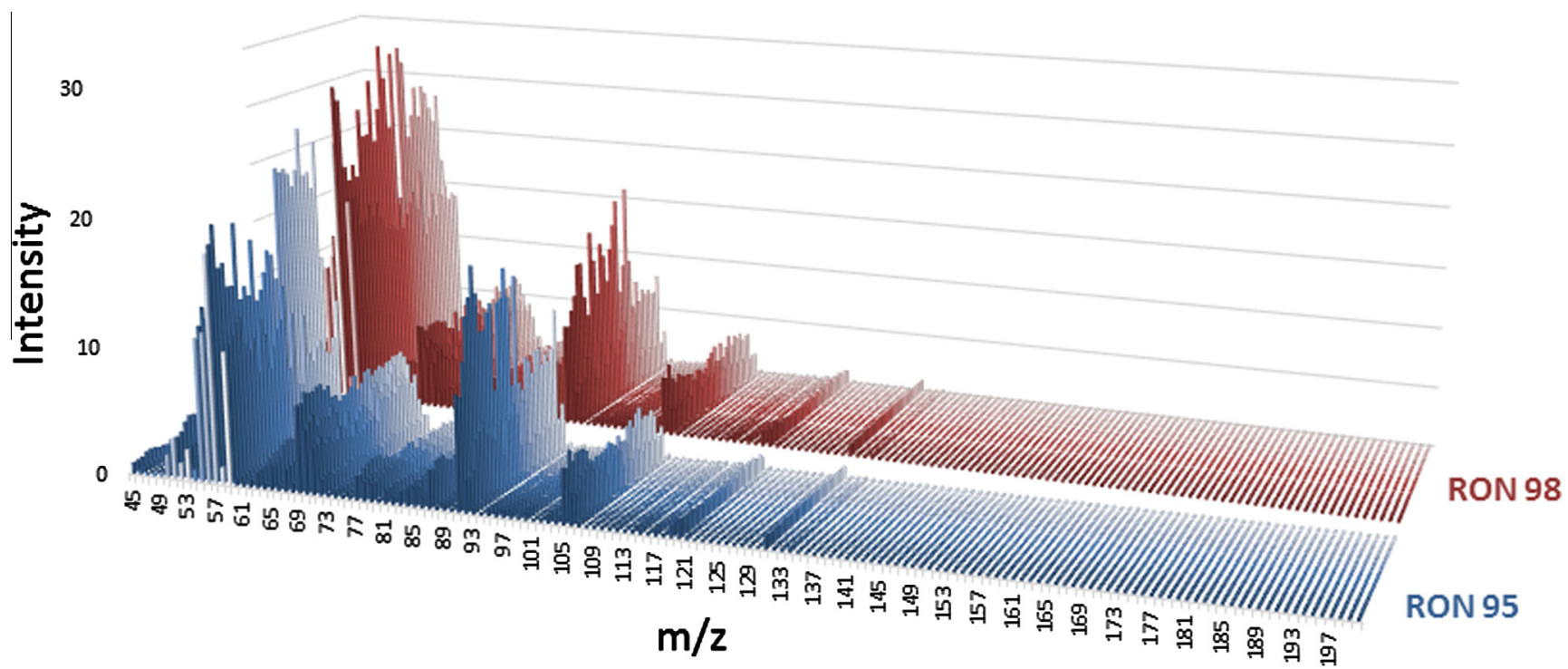

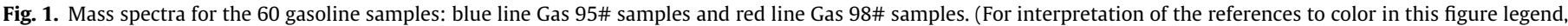
the reader is referred to the web version of this article.)

similarity (dissimilarity) at which any of the two main clusters were joined. The Ward method and square Euclidean distance were used to form clusters.

It can be seen that all of the samples were grouped into four clusters (A, B, C and D) and all samples in each cluster are from only one type of gasoline (Clusters A and B contain samples from Gas 95\# and Clusters B and C contains samples from Gas 98\#).

A primary trend for the group can be seen with the Gas 98\# samples (cluster C and D), which are separated from cluster B containing 17 samples from the Gas 95\# set. However, cluster A, which contains the remaining samples from the Gas 95\# set, is separated from the rest of the clusters to form an independent set at a higher level.

The results discussed above indicate that the data from the HSMS analyses used to perform the HCA are related to the compounds that are responsible for the discrimination of the 2 types of gasoline, even though the 155 signals obtained from the HS-MS are not sufficient to obtain a perfect separation of the analyzed samples.

Given that the non-supervised technique suggested some kind of classification for the samples based on their RON, a supervised technique, namely Linear Discriminant Analysis (LDA), was applied to the whole body of mass spectra. In order to obtain a more robust discrimination prior to running the LDA, $50 \%$ of the samples were randomly selected as a training set in order to obtain discriminant functions and the other $50 \%$ of the samples were then used as a validation set. In order to identify whether there are specific $m / z$ values in the mass spectra that are more significant than the others when classifying the gasoline according to the RON, a stepwise discriminant analysis was applied.

The resulting discriminant function allowed a full discrimination between the samples corresponding to different RONs. The resulting discrimination function was:

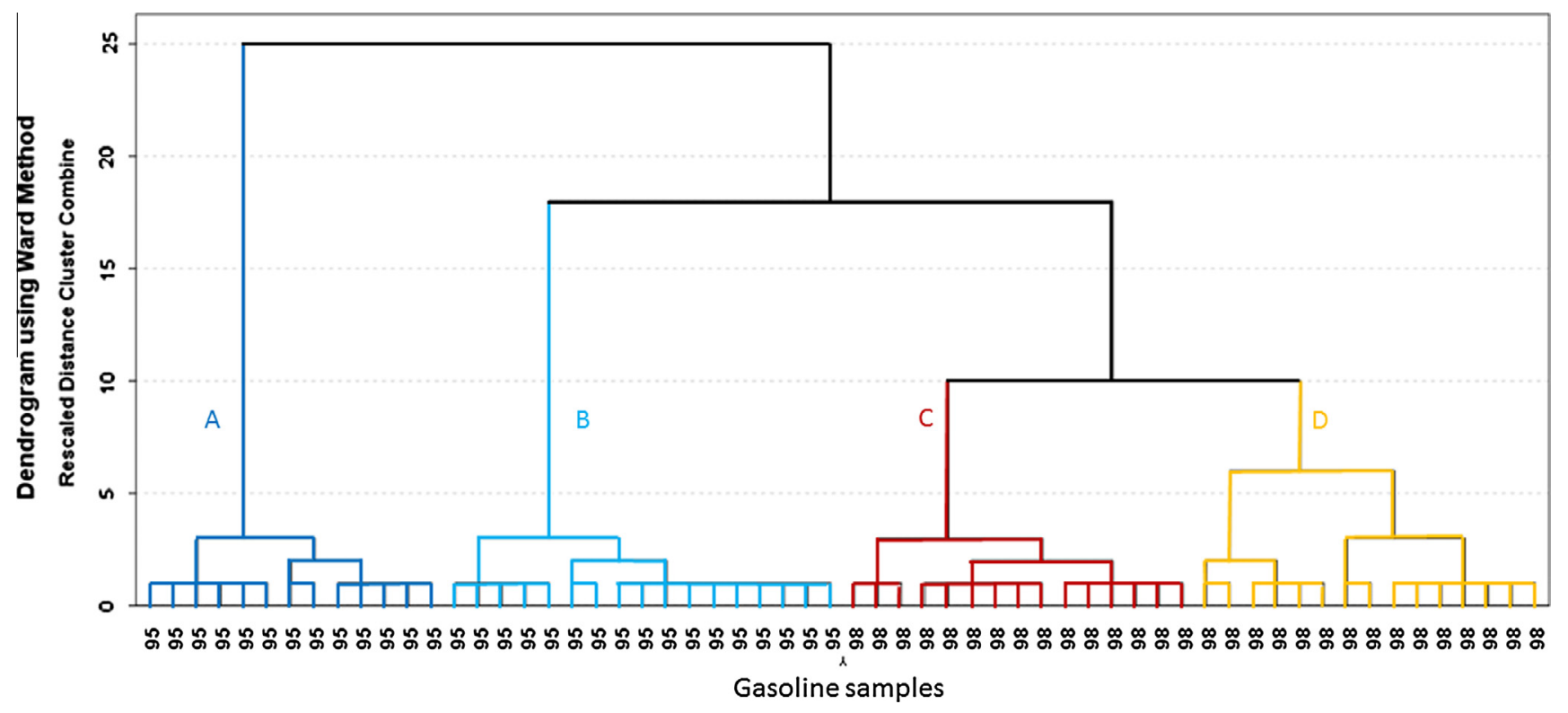

Fig. 2. Dendrogram resulting from HCA for the set of 60 gasoline samples using HS-MS data. 
$F=-2.506+6.968 * m / z(46)-1.165 * m / z(59)+10.560$

$* m / z(95)+16.819 * m / z(98)$

where $m / z(x)$ : means the signal in the mass spectrum at $m / z$ ratio $=x$.

The values obtained for both the calibration and validation set by applying the discriminant function are shown in Fig. 3. A fairly homogenous grouping can be observed for most of the samples within each group. The samples in the validation set are clearly assigned to their corresponding group without exception, i.e., $100 \%$ correct classification was obtained for samples outside the calibration set.

\subsection{NIRS chemometric study}

NIR spectroscopy is known to be an efficient tool for gasoline classification and the same set of samples were therefore analyzed by NIR in order to compare the results with those obtained by HSMS (Fig. 4).

Although there are some differences in a few areas in the ranges studied (891-1108 nm, 1233-1658 nm and 1748-1802 nm) there is no clear division of the spectra from the two types of gasoline. Once again, chemometric tools are required in order to extract the information and achieve discrimination of the samples regarding their RON.

Firstly, HCA was performed as an exploratory classification technique for gasoline by using the raw NIR data matrix. As in the previous case, the Ward method and square Euclidean distance were used to form clusters. The resulting dendrogram from this analysis is presented in Fig. 5.

Three clear clusters (A, B and C) were obtained from this HCA. However, unlike the HS-MS dendrogram, none of the clusters contain samples from only one of the gasoline types. All of the clusters have samples from both types of gasoline: Cluster A contains mainly samples from 95\# (60\%) and Cluster B from 98\# (74\%). Cluster $C$ contains samples from both sets equally.

Within cluster B there are two sub-clusters (B1 and B2); the one on the left (B1) contains 9 samples from 98\# exclusively but the one on the right (B2) has 10 samples from each type of gasoline (i.e., 5 samples from 95\# and 5 from 98\#).

Cluster $C$ joins to Clusters $A$ and $B$ at a longer distance, meaning that samples from cluster $C$ are more different than samples from $A$ and $B$, which are very similar to one another based on the NIR data.

These results indicate that it is not possible to group the samples of different types using all of the NIR data. As a consequence,

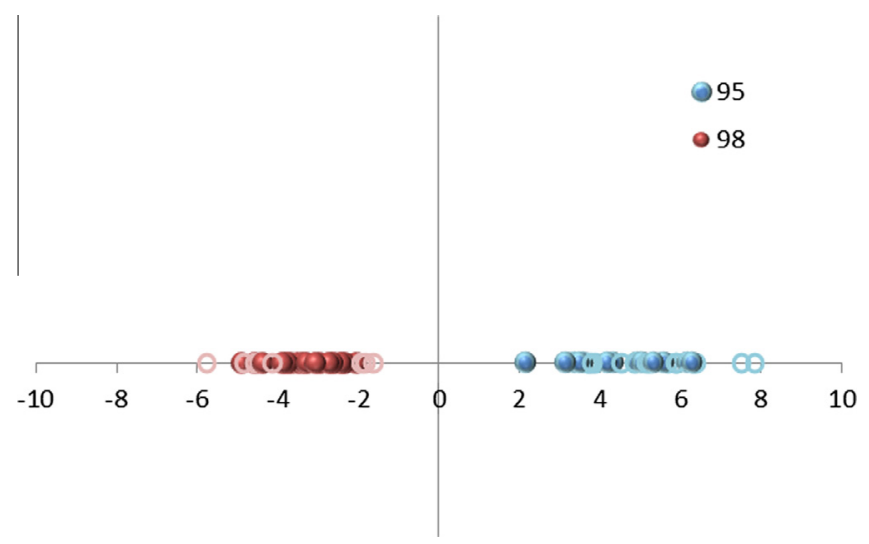

Fig. 3. Territorial map obtained in the LDA using HS-MS data. The dots represent the samples from the calibration set and circles represent the samples from the validation set.

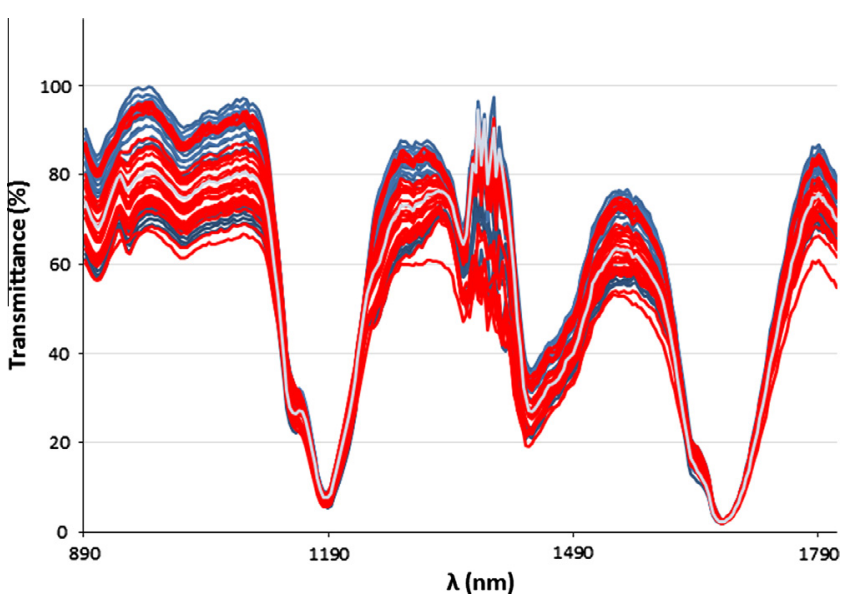

Fig. 4. NIR spectra for the 60 gasoline samples from the 2 different classes recorded in the NIR. The blue lines correspond to gas 95\# spectra and the red lines to gas 98\# spectra. (For interpretation of the references to color in this figure legend, the reader is referred to the web version of this article.)

a stepwise LDA was carried out in order to obtain not only the discrimination function, but also to check whether there are some specific NIR regions that are more significant than the others when classifying gasoline according to the RON.

A linear discriminant analysis was then carried out on the whole body of NIR spectra. The same set of samples as used for the calibration and validation sets for the HS-MS study was used to calibrate and validate the results from this analysis.

As in the previous HS-MS study, the resulting linear discriminant function allows a perfect classification (100\%) of the gasoline samples to be obtained regarding the RON for samples in the calibration set. In this case, however, a larger number of variables were needed to achieve a full discrimination. The wavelengths selected for the discrimination were $965,1410,1453,1485,1524,1573$, 1607 and $1610 \mathrm{~nm}$, and the resulting discrimination function was as follows:

$$
\begin{aligned}
F= & -87.169-1.505 * \lambda(965)+0.177 * \lambda(1410)-2.315 \\
& * \lambda(1453)+3.669 * \lambda(1485)-1.258 * \lambda(1524)+1.468 \\
& * \lambda(1573)-2.228 * \lambda(1607)+4.024 * \lambda(1610) .
\end{aligned}
$$

where $\lambda(x)$ : means the signal in the NIR spectrum at wavelength $=x \mathrm{~nm}$.

The values obtained for each gasoline sample, including both calibration and validation sets, by applying the discrimination function are shown in Fig. 6. All of the samples were discriminated by their RON and therefore the same classification results were obtained as in the previous HS-MS analysis. However, the distribution for samples from the validation set was quite different in the two cases; NIRS analysis produced a more disperse distribution for these samples whereas the HS-MS results showed a more homogenous distribution for the samples of the validation set.

Different signals and compounds were used for HS-MS and NIR spectroscopy to develop a discriminant model. In the case of HSMS, volatile compounds were analyzed while in NIR spectroscopy the sample in liquid state was analyzed, then all compounds producing signal in the NIR region were used. Both of these techniques allow a full discrimination of the gasoline samples according to the RON. 


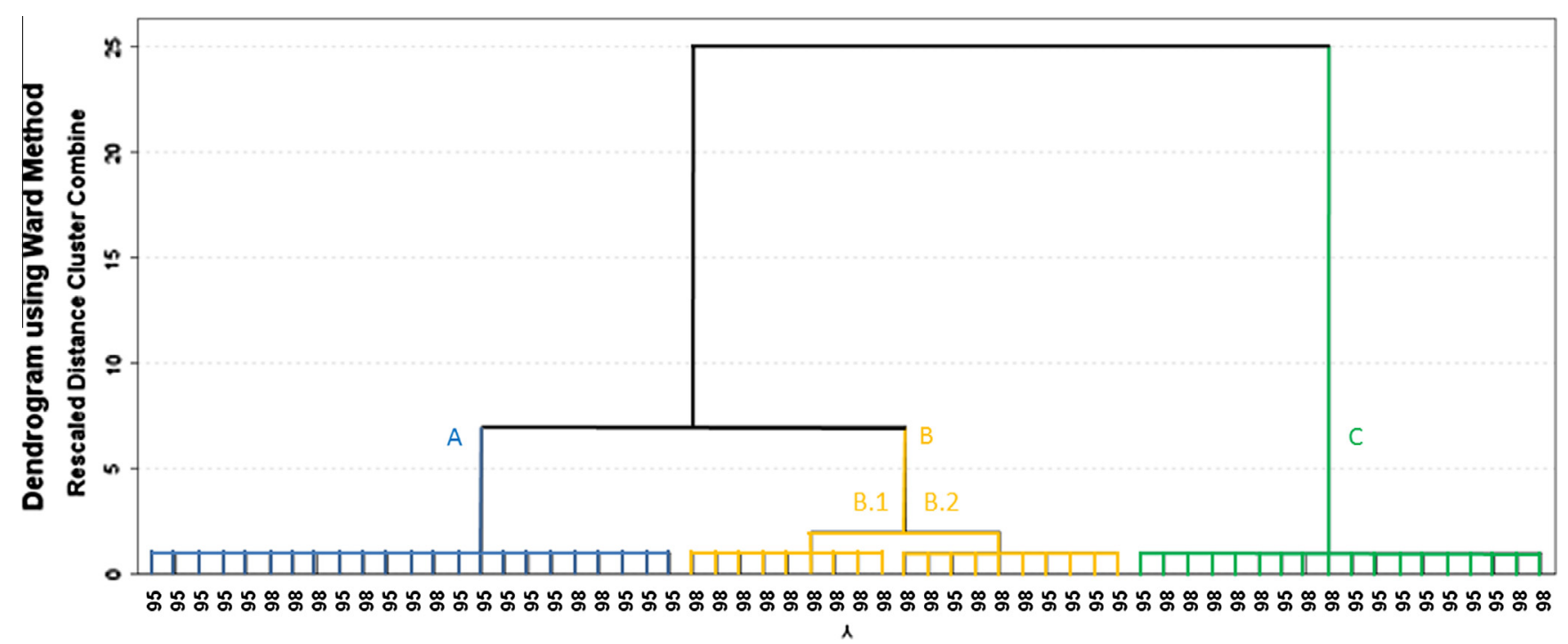

Fig. 5. Dendrogram showing the percentage similarity of 60 gasoline samples when using the NIR transmittance in the range $891-1812$ nm.

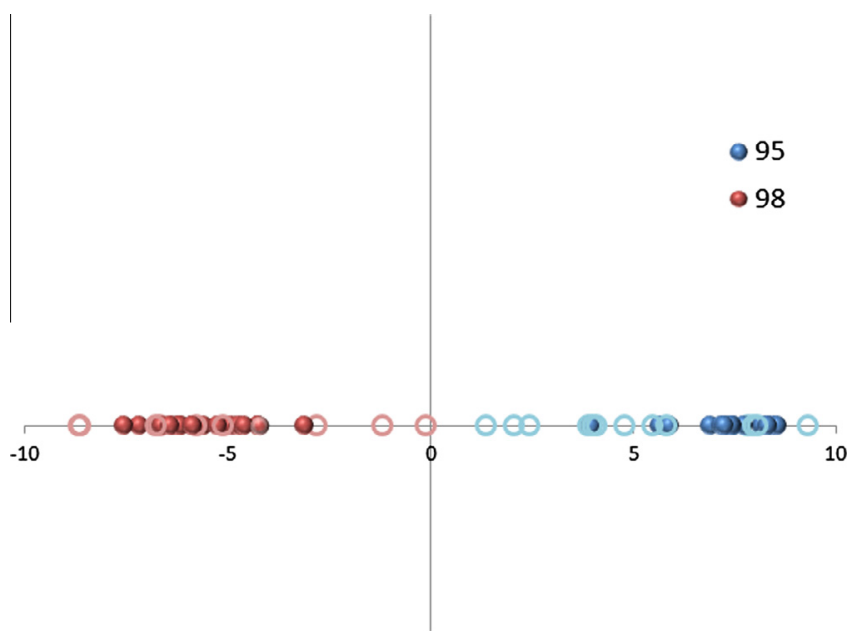

Fig. 6. Territorial map obtained in the LDA using NIRS data. The dots represent the samples from the calibration set and the circles the samples from the validation set.

\section{Conclusions}

HS-MS and NIR spectroscopy combined with chemometric tools have proven to be effective techniques for the classification of gasoline according to the RON.

HCA was able to group samples of different types into different clusters using HS-MS data but this approach was unsuccessful when NIR data were used, since more than $25 \%$ of the samples were misclassified. However, LDA allowed a full discrimination of the gasoline samples on using either of the aforementioned two techniques. Only four $m / z$ values $(46,59,95$ and 98) on using HS-MS or three NIR regions (below $1000 \mathrm{~nm}, 1400-1500 \mathrm{~nm}$ and $1600 \mathrm{~nm}$ ) are required for the discrimination of the gasoline samples.

It should be noted that HS-MS gave rise to a less disperse distribution of the samples within each type of gasoline, which indicates that the volatile compounds are more appropriate to discriminate gasoline samples with different RON than the signals from the liquid sample.

Based on these results, HS-MS has proven to be a promising tool and it can be considered as a good alternative for a general quality recognition method in refineries. Furthermore, this technique could be applied for the classification of gasoline in terms of the origin and even discrimination studies of other ignitable liquids.

\section{Acknowledgements}

MFG gratefully acknowledges the University of Cádiz for a Ph.D. studentship. Authors are very grateful to Mr. Jesús Ruiz who provided technical assistance to the HS-MS system.

\section{References}

[1] Stauffer E, Dolan JA, Newman R. Chapter 7 - Flammable and combustible liquids. In: Stauffer E, Dolan JA, Newman R, editors. Fire debris analysis. Burlington: Academic Press; 2008. p. 199-233.

[2] BOE-A-2010-13704, Real Decreto 1088/2010, de 3 de septiembre, in: T.Y.C. MINISTERIO DE INDUSTRIA (Ed.), 2010, pp. 76436-45.

[3] Standard test method for research octane number of spark-ignition engine fuel 2008 ASTM D2699-08.

[4] Doble P, Sandercock M, Du Pasquier E, Petocz P, Roux C, Dawson M. Classification of premium and regular gasoline by gas chromatography/mass spectrometry, principal component analysis and artificial neural networks. Forensic Sci Int 2003;132:26-39.

[5] Sandercock PML, Du Pasquier E. Chemical fingerprinting of unevaporated automotive gasoline samples. Forensic Sci Int 2003;134:1-10.

[6] Sandercock PML, Du Pasquier E. Chemical fingerprinting of gasoline: 2 Comparison of unevaporated and evaporated automotive gasoline samples. Forensic Sci Int 2004;140:43-59.

[7] Ignitable Liquids Reference Collection Database.

[8] Gaganis V, Pasadakis N. Characterization of oil spills in the environment using parallel factor multiway analysis. Anal Chim Acta 2006;573-574:328-32.

[9] Yuan B, Warneke C, Shao M, de Gouw JA. Interpretation of volatile organic compound measurements by proton-transfer-reaction mass spectrometry over the deepwater horizon oil spill. Int J Mass Spectrom 2014;358:43-8.

[10] Al-Ghouti MA, Al-Degs YS, Amer M. Determination of motor gasoline adulteration using FTIR spectroscopy and multivariate calibration. Talanta 2008;76:1105-12.

[11] Teixeira LSG, Oliveira FS, dos Santos HC, Cordeiro PWL, Almeida SQ Multivariate calibration in Fourier transform infrared spectrometry as a tool to detect adulterations in Brazilian gasoline. Fuel 2008;87:346-52.

[12] Ré-Poppi N, Almeida FFP, Cardoso CAL, Raposo Jr JL, Viana LH, Silva TQ, et al Screening analysis of type C Brazilian gasoline by gas chromatography - Flame ionization detector. Fuel 2009;88:418-23.

[13] Pierce KM, Hope JL, Johnson KJ, Wright BW, Synovec RE. Classification of gasoline data obtained by gas chromatography using a piecewise alignment algorithm combined with feature selection and principal component analysis. J Chromatogr A 2005;1096:101-10.

[14] Desa WNSM, Daéid NN, Ismail D, Savage K. Application of unsupervised chemometric analysis and self-organizing feature map (SOFM) for the classification of lighter fuels. Anal Chem 2010;82:6395-400. 
[15] Rudnev VA, Boichenko AP, Karnozhytskiy PV. Classification of gasoline by octane number and light gas condensate fractions by origin with using dielectric or gas-chromatographic data and chemometrics tools. Talanta 2011;84:963-70.

[16] ASTM, E1618-11 Standard test method for ignitable liquid residues in extracts from fire debris samples by gas chromatography-mass spectrometry, West Conshohocken, PA; 2011.

[17] Khanmohammadi M, Garmarudi AB, Ghasemi K, de la Guardia M. Quality based classification of gasoline samples by ATR-FTIR spectrometry using spectral feature selection with quadratic discriminant analysis. Fuel 2013;111:96-102.

[18] Balabin RM, Safieva RZ. Motor oil classification by base stock and viscosity based on near infrared (NIR) spectroscopy data. Fuel 2008;87:2745-52.

[19] Balabin RM, Safieva RZ, Lomakina EI. Near-infrared (NIR) spectroscopy for motor oil classification: From discriminant analysis to support vector machines. Microchem J 2011;98:121-8.

[20] Balabin RM, Safieva RZ. Gasoline classification by source and type based on near infrared (NIR) spectroscopy data. Fuel 2008;87:1096-101.

[21] Balabin RM, Safieva RZ, Lomakina EI. Gasoline classification using near infrared (NIR) spectroscopy data: Comparison of multivariate techniques. Anal Chim Acta 2010;671:27-35.

[22] Khanmohammadi M, Garmarudi AB, Garmarudi AB, de la Guardia M. Characterization of petroleum-based products by infrared spectroscopy and chemometrics. TrAC, Trends Anal Chem 2012;35:135-49.
[23] da Silva MPF, Brito LRe, Honorato FA, Paim APS, Pasquini C, Pimentel MF. Classification of gasoline as with or without dispersant and detergent additives using infrared spectroscopy and multivariate classification. Fuel 2014;116:151-7.

[24] Li S, Dai L-K. Classification of gasoline brand and origin by Raman spectroscopy and a novel R-weighted LSSVM algorithm. Fuel 2012;96:146-52.

[25] Sobanski T, Szczurek A, Nitsch K, Licznerski BW, Radwan W. Electronic nose applied to automotive fuel qualification. Sensor Actuator B Chem 2006;116:207-12.

[26] Balabin RM, Lomakina EI. Support vector machine regression (SVR/LS-SVM)-an alternative to neural networks (ANN) for analytical chemistry? Comparison of nonlinear methods on near infrared (NIR) spectroscopy data. Analyst 2011;136:1703-12.

[27] Balabin RM, Lomakina EI, Safieva RZ. Neural network (ANN) approach to biodiesel analysis: Analysis of biodiesel density, kinematic viscosity, methanol and water contents using near infrared (NIR) spectroscopy. Fuel 2011;90: 2007-15.

[28] Ferreiro-Gonzalez M, Ayuso J, Alvarez JA, Palma M, Barroso CG. New headspace-mass spectrometry method for the discrimination of commercial gasoline samples with different research octane numbers. Energy Fuels 2014;28:6249-54.

[29] SPSS; SPSS Science. 233 S. Wacker Drive. 11th floor; Chicago. IL 60606-6307 (SPSS Inc., Chicago. IL. USA). 